\title{
The Relationship between Crown Size and Complexity in Two Collections
}

\author{
Bridgett A. Williams and Robert S. Corruccini* \\ Department of Anthropology, Southern Illinois University, Carbondale, IL
}

ABSTRACT Many studies show human tooth crown size increases with increasing crown complexity (i.e., extra cusps, tubercles or grooves). Plio-Pleistocene hominid tooth size reduction has also incurred reduction in complexity, which plays into many theories that attempt to explain this well known, sustained odontometric reduction. We correlated various types of tooth complexity with measured tooth size in two collections: the widely used ASU dental models (238 MD and BL dimensions of 119 teeth involved in 19 post-incisor model plaques),

Beginning in the Paleolithic and continuing through the present, a Pleistocene through Holocene trend within the human dentition has been recognized. Dental reduction, a reduction in tooth crown diameter, dramatically changed the morphology of teeth. Though Europe demonstrated a dramatic form of this trend between the Early and Late Upper Paleolithic, other areas expressed this change as well, including Nubia where dental reduction occurred most dramatically from $12,000-10,000$ B.P. and 5,300-3,000 B.P. (Calcagno, 1986; Hillson, 1996). Dental reduction is a clear representation of morphological change seen within the fossil record, the cause of which has been debated.

Many scholars have attempted to explain dental reduction (Armelagos et al., 1989, Bailit and Friedlaender, 1966; Brace and Mahler, 1971; Calcagno, 1986, Calcagno and Gibson, 1988, 1991; Frayer, 1977, 1978; Smith 1982; Smith et al., 1986; Greene, 1970) without agreement. One possible explanation lies within the archaeological record. Increased tool quality during the Upper Paleolithic reduced the masticatory demand on teeth (Frayer, 1978; Brace and Mahler, 1971; Brace, 1963). Because no selective pressures existed to maintain large tooth size, reduction resulted from the Probable Mutation Effect (PME), which suggests that, "through random mutations, the developmental processes controlled by complex genetic mechanisms will be disrupted with the final result being an incomplete or simplified structure" (Brace and Mahler, 1971:192). This theory became quite controversial because of its rejection of the selection-based synthetic theory of evolution, which remains the accepted mode (Bailit and Friedlaender, 1966).

Another explanation for crown size reduction also relates to changes seen within the archaeological record (Calcagno and Gibson, 1991), changes resulting from and in Newton Plantation slave remains from Barbados (736 dimensions of 368 teeth from ca. 100 individuals consisting of 8 post-canine types: mandibular premolars and molars, and maxillary molars). Significant positive correlations show crown size and crown complexity decrease together, thus either type of data might serve to document this decline. However the degree and pattern of this positive correlation was distinct in the two samples. Dental Anthropology 20:29-32.

positive selective pressures for small teeth. As tool use reduced the functions that were placed upon the jaw, large chewing muscles and a robust jaw forms were no longer necessary. The teeth develop cryptically as a result of genetic pressures, independent from jaw size selection. The large teeth that were adaptive previous to the tool technology revolution crowded the smaller jaws. As a result of this malocclusion, dental infection and disease emerged, causing selection for smaller teeth (Calcagno and Gibson, 1991). Countless other theories exist which attempt to explain dental reduction, though debate still surrounds the trend.

Garn et al. (1966) suggest that morphologically complex (hyperodontic) tooth crowns are usually seen within larger teeth (1966). A recent study by Harris tracks one such trait, Carabelli cusp (2007). Carabelli's trait may sometimes be as large as the principal cusp of the crown, and Harris (2007) found that the greater the expression of Carabelli's trait, the larger the overall crown size of the tooth. Harris measured teeth in the living which may not reflect the trend within teeth over time in the Upper Paleolithic; however, others suggest that the reducing dentitions during the Upper Paleolithic moved toward relatively simple crown morphologies. Cucina et al., (1999) and Coppa et al. (1998) contrast gene flow versus in situ selection models for the Italian Neolithic and later stages, and Coppa et al. $(1999,2007)$ analyze dental traits changing from the Italian Paleolithic through Mesolithic and Neolithic to almost contemporary agricultural times,

*Correspondence to: Robert S. Corruccini, Department of Anthropology, Southern Illinois University, Carbondale, IL 62901-4502.

E-mail : rcorrucc@siu.edu 
but the progressively hypodontic traits they use may merely signal tooth size reduction.

To further investigate the trend of dental reduction, the present study analyzes several different dental traits and their effect on overall dental size. Our hypothesis is that hyperodontic teeth will be larger in both breadth and width than crowns showing hypodontic traits.

\section{MATERIALS AND METHODS}

Two trials were organized in order to analyze dental traits. Part A involved data concerning the ASU Dental Anthropology System (Turner et al., 1991). Part B analyzed a dental collection from Barbados on loan to the SIUC Anthropology department.

\section{Part A}

The ASU Dental Anthropology System is the primary plaster cast system for standardized grading of varying dental complexity (Turner et al., 1991). This system displays 27 different dental traits in upper and lower teeth grading the increasing complexity of a given trait from 0 to 5 in most cases, with some plaques measuring up to 9 grades of complexity for a single trait (Turner et al., 1991). The traits described within the ASU Dental Anthropology System represent traits that are easily standardized for study among many different dental specimens. Also, they are easily observed in teeth reducing potential for inter-observer error.

Of the 27 traits displayed within the system, 19 were chosen for analysis. The traits included: upper molar cusp 5, hypocone cusp 4, metacone cusp 3, parastyle, Carabelli's trait, anterior fovea lower M1, distal accessory ridge upper canine and lower canine, tuberculum dentale upper I1, bushman canine, deflecting wrinkle, protostylid, lower molar cusp 5, cusp 6, cusp 7, mid trigonid crest (lower M1), mid trigonid crest (lower M2), mesial lower premolar cusp number (" $\mathrm{p} / 1$ " plaque, or trait 16 as described with the associated sheet), and distal lower premolar cusp number (" $\mathrm{p} / 2$ " which is trait 17). The remaining 8 plaques which were not measured are related to shovel-shaped incisors and were excluded from the study because these traits are not clearly known to be present in dentitions prior to the Upper Paleolithic and do not clearly relate to increased crown complexity of the incisors.

Each tooth displayed on a given plaque was analyzed for standard maximum mesiodistal and buccolingual measurements. Two different sets of calipers, a digital model, Mitutoyo Absolute Digimatic and analog model MitutoyoNo.505-636 calibrated to $0.05 \mathrm{~mm}$ were used. The digital model was the primary set used for measurements; however the tips on this model were fairly wide and blunt (better for measuring buccolingual breadths) and could not properly mesiodistally measure some of the teeth on the plaques because of their closeness to one another. In these cases the sharpened-point analog model was used to measure the tooth. In order to ensure reliable readings for each measure, the calipers were calibrated to 0.00 before taking each measurement. Both the mesiodistal and buccolingual measurements were taken at least twice for each tooth to ensure reliable measurements were being recorded. All data were entered into a spreadsheet. Mesiodistal and buccolingual measurements were each entered on a separate line independent of each other in order to assess the significance that the crown complexity had on each tooth measurement independent of the other.

After all of the plaques were measured, we proceeded to correlate complexity compared to tooth size measurements using a collection of teeth in an actual single population.

\section{Part B}

The Department of Anthropology excavated a large collection of remains from Newton Plantation, Barbados that pertained to slaves from the island country (Corruccini and Handler et al., 1982). These remains had been analyzed previously for more traditional cusp number and tooth mesiodistal and buccolingual measurements. No detailed, highly multi-state traits were analyzed from these remains, unlike the ASU system. This grading system was much simpler, consisting of 3 or 2 cusped mandibular premolars, 5 or 4 cusped mandibular molars, and 4 or 3-cusped maxillary molars. As there was no variation to the 2-cusped mesial mandibular premolars, they were considered to be a $2+$ (entered as 2.5) when adjacent to a canine tooth with a prominent accessory ridge or tubercle. Similarly, maxillary first molars did not vary from showing the 4 basic cusps but were coded as 4.5 when clearly showing a Carabelli cusp. The original data for cusp numbers and mesiodistal and buccolingual measures were already in existence for mesial and distal lower premolars and upper and lower molars 1,2 , and 3 , so the teeth themselves were not measured for this particular study (Corruccini et al., 1982). A total of approximately 100 individuals available in the collection were analyzed. For most individuals only the measurements and cusp number for the left tooth were used. In cases where no data existed for the left, the numbers for the right tooth were collected instead. For some individuals not all premolars and molars were present to measure, in this case all available measurements for the left (if available) and the right (if not) were taken. The measures were entered into a database in the same manner as the ASU measures.

\section{RESULTS}

\section{Part A}

A total of 119 individual teeth on 19 ASU plaques were measured for both mesiodistal and buccolingual 
TABLE 1. MANOVA with tooth size (MD or BL dimension in $\mathrm{mm}$ ) as the dependent variable ${ }^{1}$

PART A: ASU Plaques

\begin{tabular}{lccccc}
\hline Source & Sum Squares & df & Mean square & F Value & P Value \\
\hline Ordinal score & 81.3 & 21 & 3.87 & 1.79 & 0.021 \\
Plaque (trait) & 139.2 & 1 & 139.2 & 64.4 & 0.000 \\
MD vs. BL & 2.1 & 1 & 2.1 & 0.981 & 0.323 \\
& & PART B: Newton Plantation & & \\
\hline Source & Sum Squares & df & Mean square & F Value & P Value \\
\hline Cusp number & 5155054 & 3 & 1718351. & 186.0 & 0.000 \\
Tooth & 1528223 & 1 & 1528223. & 64.4 & 0.000 \\
MD vs. BL & 450879 & 1 & 450879. & 49.0 & 0.000 \\
\hline
\end{tabular}

${ }^{1}$ Crown dimension codes are mesiodistal (MD) and buccolingual (BL).

dimensions, hence 238 total cases yielding 237 degrees of freedom. The overall multiple correlation between increased crown complexity and an increase in overall crown size for the 19 traits in total was 0.640 (Table 1), thus over these ASU plaques there is a significant multiple $\mathrm{R}$ between tooth size (both mesiodistal length [L] and buccolingual breadth [B]) and the combined effects of MD Length versus BL Breadth, crown complexity, and the individual plaque. The interaction of $\mathrm{MD}$ versus $\mathrm{BL}$ dimensions in fact did not contribute significantly to this; in theory either measure could be used to the exclusion of the other, and there is considerable redundancy. The contribution of heterogeneity over different plaques was quite a bit stronger than the contribution of crown complexity score, but the latter nevertheless was significantly greater than zero $(\mathrm{F}=1.79, \mathrm{P}<0.02$; even with the degrees of freedom halved to acknowledge the redundancy, $\mathrm{P}<0.03)$. Complexity-size correlations ranged above $r=+0.7$ for several plaques, but the sample size per plaque was technically very small. The pooled weighted correlation was $r=0.41$.

\section{Part B}

A total of 368 teeth from the Newton Plantation population were analyzed for buccolingual and mesiodistal dimensions. In this much larger (but probably much more redundant) sample of teeth, there was a very significant multiple $R=0.792$ between size on the one hand and complexity, tooth type, and Lversus- $B$, with the latter factor relatively small $(F=48)$ but significant, meaning mesiodistal and buccolingual dimensions offered a significantly large amount of independent information. The complexity contribution to the multiple R (unlike Part A above) is slightly larger than the tooth type contribution and is highly significant even when drastically reducing the degrees of freedom.
Partial correlation (holding tooth type constant) between cusp number and size was $r=+0.639$.

\section{DISCUSSION}

Thus in different ways, the two data sets indicate highly significant positive correlation between hyperodontic aspects of crown complexity and larger crown size.

Dental reduction is a trend that baffles many researchers. Within early (Pliocene) hominid history, teeth were selected to become larger in order to combat effects of attrition; however, within the Paleolithic and Mesolithic the dramatic change to smaller teeth contradicts the previous evolution of the dentition. To explain this trend, which is clearly seen in the fossil record, several researchers have proposed similar ideas. Harris (2007) shows Carabelli's cusp has more complex expression when the overall diameter of the tooth crown becomes larger. Garn et al. (1966) found that mesiodistal crown diameter is significantly related to cusp number, with larger teeth in general possessing more cusps. That human teeth became not only smaller but less complex is not inconsistent with various theories, for instance those highlighting body size, caries resistance, and PME.

With the present study, attempts have been made to establish what relationship exists between crown complexity and overall mesiodistal and buccolingual diameters. Results from the ASU system suggest that there is indeed a positive correlation between morphologically complex teeth and large tooth size across different teeth and traits. There is admittedly some inconsistency with measurements of the ASU dental system because these plaques were created with ordinal examples of crown complexity, and there was no alternative to correlating ordinal with continuous data.

The Newton plantation data allowed independent tracking of the correlation between crown complexity 
and tooth size in a relatively homogeneous population. These data repeat the positive correlation between crown complexity and larger teeth.

This study does indicate a significantly strong relationship between increased crown complexity and larger teeth; however, more research should be conducted, especially with populations from the Paleolithic to determine if this relationship is seen to the same extent over time within the fossil record. Until contradicting evidence emerges, it may be seen as equivalently valid to measure teeth or to use nonmetric traits for genetic purposes. It is also pointed out that buccolingual tooth dimensions are much more standardized, and much more resistant to change from attrition than other traits (Hill, 2004).

\section{LITERATURE CITED}

ArmelagosGJ, VanGervenDP, GoodmanAH, CalcagnoJM. 1989. Post Pleistocene facial reduction, biomechanics and selection against morphologically complex teeth: a rejoinder to Macchiarelli and Bondioli. Hum Evol 4:1-7.

Bailit HL, Friedlaender JS. 1966. Tooth size reduction: a hominid trend. Am Anthropol 68:665-672.

Brace CL. 1963. Structural reduction in evolution. Am Nat 97:39-59.

Brace CL, Mahler PE. 1971. Post-Pleistocene changes in the human dentition. Am J Phys Anthropol 34:191204.

Calcagno JM. 1986. Dental reduction in post-Pleistocene Nubia. Am J Phys Anthropol 70:349-363.

Calcagno JM, Gibson KR. 1988. Human dental reduction: natural selection or the probable mutation effect. Am J Phys Anthropol 77:505-517.

Calcagno JM, Gibson KR. 1991. Selective compromise: evolutionary trends and mechanisms in hominid tooth size. In: Kelley MA, Larsen CS, editor, Advances in dental anthropology. New York: Wiley Liss. p 5976.

Corruccini RS, Handler JS, Mutaw RJ, Lange FW. 1982. Osteology of a slave burial population from Barbados, West Indies. Am J Phys Anthropol 59:443-459.

Coppa A, Cucina A, Lucci M, Vargiu R, Mancinelli D. 2007. Origins and spread of agriculture in Italy: a nonmetric dental analysis. Am J Phys Anthropol 133:918-930.
Coppa A, Cucina A, Mancinelli D, Vargiu R, Calcagno J. 1998. Dental anthropology of central-southern Iron Age Italy: the evidence of metric versus non-metric traits. Am J Phys Anthropol 107:371-386.

Coppa A, Cucina A, Vargiu R, Mancinelli D, Lucci M. 1999. The Pleistocene-Holocene transition in Italy: the contribution of the morphological dental traits. Am J Phys Anthropol Suppl 28:111.

Cucina A, Lucci M, Vargiu R, Coppa A. 1999. Dental evidence of biological affinity and life conditions of prehistoric Trentino (Italy) samples from the Neolithic to the Early Bronze Age. Int J Osteoarchaeol 6:404416.

Frayer DW. 1977. Metric dental change in the European Upper Paleolithic and Mesolithic. Am J Phys Anthropol 46:109-120.

Frayer DW. 1978. The Evolution of the Dentition in Upper Paleolithic and Mesolithic Europe. Lawrence: University of Kansas Publications in Anthropology, no. 10.

Garn SM, Lewis AB, Dahlberg AA, Kerewsky RS. 1966. Interaction between relative molar size and relative number of cusps. J Dent Res 45:1240.

Greene DL. 1970. Environmental influences on Pleistocene hominid dental evolution. BioScience 20:276-279.

Harris EF. 2007. Carabelli's trait and tooth size of human maxillary first molars. Am J Phys Anthropol 132:238246.

Hill MK. 2004. Dental reduction and diet in the prehistoric Ohio River Valley. Dent Anthropology 17: 34-44.

Hillson S. 1996. Dental anthropology. Cambridge: Cambridge University Press.

Smith P. 1982. Dental reduction: selection or drift? In: Kurten B, editor. Teeth: Form, function, and evolution. New York: Columbia University Press, p 366-379.

Smith P, Wax Y, Adler F, Silberman U, Heinic G. 1986. Post-Pleistocene changes in tooth root and jaw relationships. Am J Phys Anthropol 70:339-348.

Turner II CG, Nichol CR, and Scott GR. 1991. Scoring procedures for key morphological traits of the permanent dentition: The Arizona State University dental anthropology system. In: Kelley MA, Larsen CS, editors, Advances in dental anthropology. New York: Wiley \& Sons. p 13-31. 